\title{
Strategy to validate sensor-placement methodologies in the context of sparse measurement in complex urban systems
}

\author{
Numa J. Bertola \\ ETH-Zurich, Future Cities \\ Laboratory. Singapore-ETH Centre, \\ Singapore and EPFL - Swiss \\ Federal Institute of Technology \\ numa.bertola@epfl.ch
}

\author{
Alberto Costa \\ ETH-Zurich, Future Resilient \\ Systems \\ Singapore-ETH Centre, Singapore \\ costa@lix.polytechnique.fr
}

\author{
Ian F.C. Smith \\ EPFL - Swiss Federal Institute of \\ Technology. Applied Computing \\ and Mechanics Laboratory \\ (IMAC). Lausanne, Switzerland \\ ian.smith@epfl.ch
}

\begin{abstract}
The Internet of Things creates opportunities to develop data-driven design methodologies for smart cities. However, effects rather than causes are often measured in complex urban systems, requiring robust data-interpretation methodologies. Additionally, effective monitoring of large urban components, such as civil infrastructure, often involves multiple sensor devices and invasive sensor systems. In these situations, the design of measurement systems is an important task. Usually, this task is carried out by engineers using only qualitative rules of thumb and experience. Recently, researchers have developed quantitative sensor-placement methodologies to maximize the information gain of measurement systems. Nonetheless, these methodologies are only weakly validated using field measurements due to the small amount of data collected and the difficulties comparing the predicted information gain with observations. This paper proposes a validation strategy for sensor-placement methodologies. In this strategy, predictions of both individual sensor and sensor-configuration performances are compared with observations using statistical tests and hypothesis testing. The validation procedure is illustrated through three full-scale-bridge case studies. This strategy helps engineers select an appropriate methodology to design measurement systems in order to optimize data collection using sensors.
\end{abstract}

Keywords - Joint entropy, model updating, sensor placement, sparse measurements.

\section{INTRODUCTION}

The Internet of Things (IoT) involves connecting devices, objects, and people through exchanging data and information. However, when scaling up the IoT to a city level, "Things" become more complicated than simple household items.

When complex systems, such as civil infrastructure, are monitored, several challenges arise. First, the interpretation of collected data requires population-based methodologies to infer causes from effects and then predict future behavior [1]. In such situations, model-free signal-analysis approaches do not provide good support for critical decisions [2]. Additionally, the uncertainty magnitudes are much larger than in other fields as these systems are affected by their environment and because they are designed primarily to be safe rather than efficient. Behavior models produce only approximate predictions. Eventually, when "Things" must be upgraded due to a change in demand, a simple replacement should be the last option. More sustainable solutions often involve retrofitting and improvement actions rather than replacement. Such actions need to be well designed to control costs and minimize the impact on users using updated behavior models.

To illustrate these ideas, bridge infrastructure is taken as an example in this study. However, similar methodologies can be applied to a wide range of challenges that involve sensing. Many countries realize that they need to use more efficiently existing infrastructure and extend their lifespan due to the economic, environmental and social costs of replacement. Fortunately, most bridges have as-built load-carrying capacity that is well beyond that required by design standards. However, the quantification of this reserve capacity is challenging as it is affected by service-loading changes (for example, truck-load increases) ageing processes (for example, corrosion), new requirements (for example, widening bridges) and climate change. As critical limit states may involve plastic collapse, data interpretation and prediction are non-trivial. Monitoring has the potential to reveal this untapped reserve capacity, leading to important economies in asset management and smarter civil infrastructure [3]. Field measurements associated with robust data-interpretation methodologies contribute by improving the accuracy of behavior predictions, and this task is called structural identification.

The aim of structural identification is to improve knowledge of structural behaviour using field measurements. However, this task is challenging due to the unavoidable large magnitudes of uncertainties in civil infrastructure. Most studies of structural identification have used either a residual-minimization strategy or the traditional Bayesian model-updating (BMU) framework. While researchers usually assume that uncertainties have zeromean independent Gaussian forms [4], [5], these assumptions are seldom valid in the context of civil infrastructure as models are approximate and safe [6]. When systematic uncertainty is 
admitted, the formulation of BMU becomes complex and diagnosis is computationally expensive [7].

Error-domain model falsification (EDMF) [8] is an easy-touse structural-identification methodology. Model-parameter values are identified without the requirement of assumptions on uncertainty correlations between sensor locations. In this methodology, model-instance predictions are compared with field measurements at each sensor location independently. Model instances that are compatible with behavior measurements - called candidate models - are identified among an initial population, generated according to prior knowledge and engineering judgment. A model instance is rejected if residuals between predictions and measurements exceed threshold bounds for at least one sensor location. These bounds are calculated based on the modeling and measurement uncertainties and a target reliability of identification. Model instances having residuals within these bounds are included in the candidate-model set (CMS). Identified parameter values correspond to the range of values in the CMS.

Engineers usually design measurement systems using qualitative rules of thumb and signal-to-noise ratios. Quantitative studies on optimal sensor placement have been recently carried out to maximize the information collected during monitoring, where the task is usually formulated as a discrete optimization problem [9]. Additionally, sensorplacement algorithms are useful measurement-selection tools to choose most informative sensor measurements when the datainterpretation task requires selecting good data within the IoT [10]. Therefore, measurement-point-selection algorithms have the potential to be critical tools for the design of future smart cities.

The number of possible sensor configurations increases exponentially with the number of sensors and locations. However, all possible configurations cannot be assessed in a reasonable time as the sensor-placement objective functions are computationally expensive to evaluate. Therefore, most studies have used greedy algorithms to reduce the computational effort [11]. The objective function is defined in order to select sensor locations based on their predicted information content calculated for instance using information entropy in posterior model-parameter distributions [12], [13]. However, this objective function leads to a sensor-location clustering [14], limiting the total information gain. Joint entropy, evaluated using a hierarchical algorithm, is a new objective function [15] that explicitly includes mutual information between locations during sensor placement.

Sensor-placement methodologies have been only weakly validated using field measurements [16]. Many studies, see for example [17], [18] among others, only use information-gain metrics prior to monitoring to justify their sensor-placement methodologies. To the best of authors' knowledge, no methodology exists to validate sensor networks after field measurements are collected. One challenge for the validation of sensor-placement methodologies is that information-entropy and joint-entropy metrics provide only an average evaluation of the information gain at a sensor location with respect to all possible monitoring scenarios, since measurements are unknown before monitoring. Additionally, bridge load testing usually involves only a few sensors due to their costs, limiting the number of data points for each case study. Sensor data are also correlated as they measure structural system behavior; however, the correlation value between predictions at sensor locations is unknown in practice. Therefore, several case studies are necessary to validate sensor-placement methodologies as well as several assessment metrics to overcome the challenge of validation using small-data sets.

This paper introduces a stepwise process to validate sensorplacement methodologies when only sparse data sets of field measurements are available. Predicted information gains, measured in terms of information entropy of individual sensors and joint entropy of sensor configurations are compared with the observed information gain calculated as the falsification rate of model instances using EDMF. This methodology supports engineers in the choice of the sensor-placement methodology that maximizes the information gain collected by the IoT.

The paper is organized as follow. Section II contains background information on EDMF and the sensor-placement methodology. Section III presents the methodology to validate the sensor placement using sparse field measurements. Then, three case-studies are employed in Sections IV and V to validate sensor-performance predictions obtained using the hierarchical algorithm and signal-to-noise ratios respectively.

\section{BACKGROUND}

\section{A. Model-updating methodology}

EDMF [8] helps identify plausible parameter values of behavior models using information provided by field measurements. An initial population of model instances (IMS) is generated through assigning parameter values to the model class using a sampling technique, for example, Latin Hypercube.

Let $n_{y}$ be the number of measurement locations. For each location $i \in\left\{1, \ldots, n_{y}\right\}, R_{i}$ denotes the real responses of a structure (unknown in practice) and $y_{i}$ corresponds to the measured value at location $i$ that is compared to model-instance predictions $g_{i}(\boldsymbol{\theta})$. Model-prediction $U_{i, g}$ and measurement uncertainties $U_{i, y}$ are unavoidable. $U_{i, y}$ is usually estimated by conducting multiple series of tests under site conditions and based on manufacturer specifications, while $U_{i, g}$ is estimated using values taken from the literature, stochastic methods and engineering judgment. The relationship between $R_{i}, y_{i}$, and $g_{i}(\boldsymbol{\theta})$ is presented in (1).

$$
g_{i}(\boldsymbol{\theta})+U_{i, g}=R_{i}=y_{i}+U_{i, y} \forall i \in\left\{1, \ldots, n_{y}\right\} .
$$

By rearranging the terms in (1) and combining the two sources of uncertainty $U_{i, g}$ and $U_{i, y}$ in a unique source $U_{i, c}$, Eq. (2) is obtained.

$$
g_{i}(\boldsymbol{\theta})-y_{i}=U_{i, c} \forall i \in\left\{1, \ldots, n_{y}\right\} .
$$

The left-hand side of (2) shows the difference between a model-instance prediction and a field measurement at location $i$, called the residual $r_{i}=g_{i}(\boldsymbol{\theta})-y_{i}$. At each sensor location, falsification thresholds are defined in the uncertainty domain according to a confidence level. This confidence level is a tradeoff between accepting incorrect models and falsifying the 
correct model. This confidence level is typically fixed at $95 \%$ [3], [8], similarly to characteristic values for material properties in civil engineering. If the residual exceeds thresholds at one location, the model instance is falsified. On the contrary, models for which residuals are within threshold bounds at each sensor location are included in the candidate model set (CMS) and these models are used for predictions.

\section{B. Hierarchical algorithm}

In this section, the hierarchical algorithm for sensor placement is presented. The optimal measurement system depends on the goal of monitoring, such as structural identification or damage detection. Based on this choice, a behavior model is then built and possible ranges of modelparameters values are determined. For a sensor-placement methodology, inputs at each possible sensor location are a population of model predictions and the quantification of uncertainty sources.

Sensor placement is defined as an optimization task, where an objective function and optimization algorithm must be defined. The information entropy was introduced by Papadimitriou [12] as an objective function for the task of sensor placement. In EDMF, information entropy is evaluated in the prediction domain [19]. The distribution of modelinstance predictions at each sensor location $i$ is divided in intervals based on the combined uncertainty $U_{i, c}(2)$. The probability that model instance output $g_{i, j}$ falls inside the $j_{t h}$ interval among $N_{I, i}$ intervals is equal to $P\left(g_{i, j}\right)=$ $m_{i, j} / \sum_{l=1}^{N_{I, i}} m_{i, l}$, where $m_{i, j}$ is the number of model instances in the $j_{t h}$ interval. The information entropy $H\left(g_{i}\right)$ is evaluated for a sensor location $i$ according to (3).

$$
H\left(g_{i}\right)=-\sum_{j=1}^{N_{I, i}} P\left(g_{i, j}\right) \log _{2} P\left(g_{i, j}\right) .
$$

To explicitly account for the mutual information between sensor locations, joint entropy was proposed as a new sensorplacement objective function [15]. This metric evaluates the information entropy between sets of predictions while taking into account the mutual information between them. For a set of two sensors $\mathrm{i}$ and $\mathrm{i}+1$, the joint entropy $H\left(g_{i, i+1}\right)$ is defined following (4), where $P\left(g_{i, j}, g_{i+1, k}\right)$ is the probability that a model instance falls in both intervals $\mathrm{j}$ and $\mathrm{k}$ and $k \in\left\{1, \ldots, N_{I, i+1}\right\}, N_{I, i+1}$ is the maximum number of prediction intervals at location $i+1$, $i+1 \in\left\{1, \ldots, n_{s}\right\}$, and $\mathrm{n}_{\mathrm{s}}$ is the number of potential sensor locations.

$$
H\left(g_{i, i+1}\right)=-\sum_{k=1}^{N_{I, i+1}} \sum_{j=1}^{N_{I, i}} P\left(g_{i, j}, g_{i+1, k}\right) \log _{2} P\left(g_{i, j}, g_{i+1, k}\right) .(4)
$$

The joint entropy is less than or equal to the sum of the individual information entropies of sets of predictions. Eq. (5)

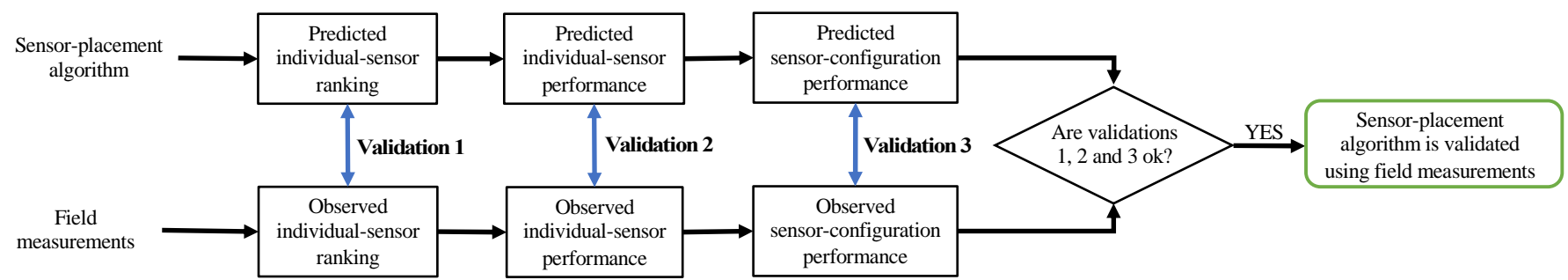

Fig. 1. Flowchart of the 3-step methodology to validate a sensor-placement algorithm using field measurements. presents the joint entropy of two sensors, where $I$ is the mutual information between sensor $i$ and $i+1$.

$$
H\left(g_{i, i+1}\right)=H\left(g_{i}\right)+H\left(g_{i+1}\right)-I\left(g_{i, i+1}\right) .
$$

Due to the large number of combinations of possible sensor configurations, an optimization strategy is required to find high-quality solutions in a reasonable amount of time. The hierarchical algorithm is a sequential algorithm with a forward approach. In a sequential search, once a sensor location is selected, this choice is not reevaluated in subsequent sensor placements. This strategy reduces the computational time and was shown to provide high-quality sensor configurations for structural identification [20].

\section{STRATEGY TO VALIDATE SENSOR-PLACEMENT METHODOLOGIES}

In this section, a strategy to compare predictions in terms of sensor-configuration performance with the observations is presented. In order to validate sensor-placement methodologies, several steps must be performed due to the small size of data sets. Sensor-placement methodologies provide three outputs that must be validated using field measurements (Fig. 1). The validation of the sensor-placement methodology is effective only if these three validation assessments are successful.

In order to select the most informative sensor locations, the sensor-placement algorithm must be able to correctly rank the sensor locations according to their performance. This sensor ranking must include locations from several sensor types such as strain gauges or deflection targets. Then, the predicted information-gain value of individual sensors must be corroborated using the observed information gain after field measurements. Finally, the total information gain of a sensor configuration must be accurately estimated by the sensorplacement algorithm in order to correctly predict the optimal

Each validation assessment is discussed in more detail below. In these assessments, the hierarchical algorithm is used as an example of sensor-placement methodology to predict sensor performance, while EDMF is used as the structuralidentification methodology to assess the observed sensor performance once field measurements are collected. Nevertheless, these assessments can be performed for any sensor-placement and data-interpretation methodologies as only normalized predicted and observed performances are compared. number of sensors that should be used for monitoring. 


\section{A. Individual sensor ranking}

The hierarchical algorithm provides an evaluation of sensor location in terms of entropy values (predicted performance), while observations are made in terms of number of falsified model instances (observed performance), called falsification rate. Although these two values cannot be directly compared, one aspect for validation is that the sensor ranking provided by the sensor-placement algorithm must be comparable with the observed ranking according to their sensor falsification performance. The sensor-placement methodology must thus select in the first position the most powerful sensors defined using falsification rate.

The first assessment involves the comparison of these sensor rankings. For each case study, sensors are ranked according to predicted and observed performance. Then, these rankings are compared using the generalized rank correlation metric $\Gamma$ [21], where $a_{i}$ denotes the information entropy and $b_{i}$ indicates the falsification performance of sensor $i$ after monitoring (6). $b_{i}$ is calculated as the proportion of model instances that are falsified by the individual field measurement $y_{i}$ using (2). When $\Gamma$ is equal to 1 , this result reflects a perfect correlation between the prediction and observation, while $\Gamma$ equal to 0 indicates no correlation between data sets. Therefore, $\Gamma$ values larger than 0.95 are required.

$$
\Gamma=\frac{\sum_{i=1}^{n} a_{i} b_{i}}{\sqrt{\sum_{i=1}^{n} a_{i}^{2}} \sqrt{\sum_{i=1}^{n} b_{i}^{2}}} .
$$

\section{B. Correspondence between predicted and observed individual-sensor performance}

An additional comparison between these data sets is aimed at evaluating the relation between the predicted (entropy value) and the observed (falsification rate) performances. Once normalized using the maximum performance value for each case study, results of all case studies can be combined. Statistical tests are performed to assess the hypothesis of a correlation between predictions of sensor performance and the observations. In case of perfect correlation, results must be close to the linear regression of $\widehat{H}=\widehat{F}$, where $\widehat{H}$ is the normalized sensor information entropy and $\hat{F}$ is the normalized falsification rate. To corroborate this assumption, the experimental linear regression must be similar to $\mathrm{x}=\mathrm{y}$ and the $R_{2}$ value must be close to 1 . Additionally, the $p$-value $(\alpha)$ is used to quantify the statistical significance of the hypothesis of a correlation between entropy and falsification performance. In statistical hypothesis testing, the p-value is defined as the probability that the data would be at least as extreme as those observed if the null hypothesis (i.e., no correlation) is true. In this study, a threshold of $\alpha$ equal to 0.05 is set to corroborate the statistical significance of the hypothesis. In other words, if $\mathrm{p}$-value is less than $\alpha$ the null hypothesis is rejected.

\section{Correspondence between predicted and observed sensor- configuration performance}

The information gain of a sensor configuration is influenced by the mutual information between sensor locations. In the hierarchical algorithm, the joint entropy assesses (4) the predicted information gain of a sensor configuration. As it explicitly accounts for the mutual information between sensor locations [15], this metric provides an output that can be corroborated using field measurements.

First, the joint-entropy assessment with respect to the number of sensors provides the information on the optimal number of sensors as usually the joint-entropy value reaches a maximum value. As the predicted information gain is assessed considering all potential field measurements, the falsification rate should not increase once the joint entropy reaches a maximum value (the inverse is not necessarily true).

Then, the predicted redundancy of information gain between two sensors must be similar to the observed mutual information. The unique information provided by sensor $i$ when this sensor is added to a configuration involving previously a given number of sensors $t(t \geq 1)$ must be correctly estimated by the sensor-placement algorithm. This new information is evaluated using the ratio between the increase of performance (joint entropy or falsification) when sensor $i$ is included in the sensor configuration $t, \operatorname{Per} f_{t+i}-\operatorname{Perf} f_{t}$, and the individual performance of sensor $i, \operatorname{Perf} f_{i}$. However, the unique information provided by sensor $i$ is influenced by the information already gained by the sensor configuration $\operatorname{Per} f_{t}$, as new information is more difficult to collect when most information has already been gained. For this reason, predicted and observed performance in terms of new information cannot be directly compared. A metric evaluating the new information is calculated in (7). The unique information is multiplied by the ratio between the information already collected by the sensor configuration, $\operatorname{Perf} f_{t}$, and the maximum theoretical information gain, $\operatorname{Per} f_{\max }$.

$\operatorname{Perf} f_{\text {max }}$ corresponds to a CMS with only a single model instance (perfect identification). In the falsification domain, $\operatorname{Per} f_{\text {max }}$ is equal to $(I M S-1) / I M S$ where $I M S$ is the initial number of model instances generated. In the joint-entropy domain, $\operatorname{Perf} f_{\text {max }}$ is equal to $-\log (1 / I M S)$ that is the maximum joint-entropy value. For both prediction and observation domains, $\operatorname{Per} f_{\text {max }}$ is thus only related to the initial population of model instances.

$$
\mathrm{N}(\mathrm{i})=\left\{\begin{array}{c}
1, \text { if } t=1 \\
\frac{\operatorname{Perf}_{t+i}-\operatorname{Perf}_{t}}{\operatorname{Perf}_{i}} * \frac{\operatorname{Perf} f_{t}}{\operatorname{Perf}_{\max }} \forall t>1
\end{array}\right.
$$

This metric allows a comparison of the predicted $\left(N_{\text {entropy }}\right)$ and observed $\left(N_{\text {fals }}\right)$ new information when sensor $i$ is added to the sensor configuration. Results of the sensor-placement methodology are corroborated only if there is a correlation between $N_{\text {fals }}$ and $N_{\text {entropy }}$. To verify this assumption, the linear regression must be similar to $\mathrm{x}=\mathrm{y}$ with $\mathrm{R}_{2}$ close to 1 . Additionally, a threshold $\alpha$ equal to 0.05 is set on the $p$-value in order to corroborate the statistical significance of the hypothesis of a correlation between the predicted and observed new information $N(i)$ given by sensor $i$ related to a sensor configuration $t$. 
TABLE I

CASE STUDY DESCRIPTION

\begin{tabular}{|c|c|c|}
\hline Parameters & Initial range & Identification \\
\hline 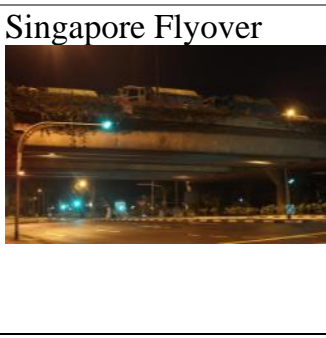 & $\begin{array}{l}4 \text { precast prestressed } \\
\text { concrete beams } \\
\text { Poured-concrete deck } \\
\text { Built in } 1985 \\
\text { Single span of } 32.0 \mathrm{~m}\end{array}$ & $\begin{array}{l}4 \text { deflection and } 2 \text { inclination measurements under a static load test. } \\
4 \text { frequency measurements under dynamic tests using } 10 \text { accelerometers. } \\
\text { Values of model parameters to identify: } \\
\text { - equivalent concrete Young's modulus } \theta_{1,1} \\
\text { - equivalent concrete density } \theta_{1,2} \\
\text { - rotational stiffness of bearing devices } \theta_{1,3} \\
\text { - vertical stiffness of bearing devices } \theta_{1,4} \\
\text { Number of initial model instances }\left(I M S_{1}\right): 1000\end{array}$ \\
\hline Exeter Bascule Bridge & $\begin{array}{l}2 \text { steel girders } \\
\text { Aluminum deck } \\
\text { Built in } 1972 \\
\text { Single span of } 17.3 \mathrm{~m}\end{array}$ & $\begin{array}{l}1 \text { deflection and } 5 \text { strain measurements under a static load test. } \\
\text { Values of model parameters to identify: } \\
\text { - equivalent deck Young's modulus } \theta_{2,1} \\
\text { - rotational stiffness of bearing devices } \theta_{2,2} \\
\text { - axial stiffness of hydraulic jacks } \theta_{2,3} \\
\text { Number of initial model instances }\left(I M S_{2}\right): 3000\end{array}$ \\
\hline 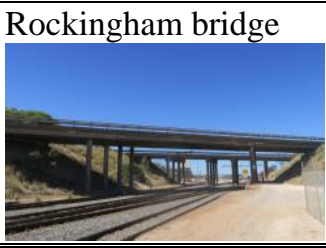 & $\begin{array}{l}8 \text { precast prestressed } \\
\text { concrete beams } \\
\text { Poured-concrete deck } \\
\text { Built in } 1970 \\
\text { Spans of } 13.7,24.3 \\
\text { and } 13.2 \mathrm{~m} .\end{array}$ & $\begin{array}{l}5 \text { strain measurements under a static load test } \\
\text { Values of model parameters to identify: } \\
\text { - beam concrete Young's modulus } \theta_{3,1} \\
\text { - deck concrete Young's modulus } \theta_{3,2} \\
\text { Number of initial model instances }\left(I M S_{3}\right): 300\end{array}$ \\
\hline
\end{tabular}

\section{CASE STUDIES - HIERARCHICAL ALGORITHM}

In this section, results of the hierarchical-algorithm sensor placement are compared with falsification performance of sensors and sensor configurations for three full-scale case studies.

Table I presents characteristics of the Singapore Flyover (Singapore) [22], [23], Exeter Bascule Bridge (UK) [24], [25] and Rockingham Bridge (Australia) [26]. Monitoring has been performed in 2016, 2017 and 2014 respectively. For each bridge, the aim of the monitoring is to identify model-parameter values in order to provide more accurate predictions of structural behavior and assessing their reserve capacity

Fig. 2. presents the sensor configuration selected by engineers for each bridge. In each case study, the sensor locations have been selected based on their predicted signal-tonoise ratio and engineering experience. In the Singapore flyover (Fig. 2A), 10 accelerometers are disposed in order to estimate the first four natural frequencies of the bridge during dynamic excitations. Therefore, these sensor locations are not directly assessed in this study as only the natural frequencies are used during falsification.

Table II presents the initial intervals of model parameters that must be identified during monitoring. These intervals are estimated based on engineering judgement of plausible parameter values for existing bridges [22-26]. For each bridge, a population of model instances IMS is generated, where each instance had a unique set of parameter values within initial feasible ranges. These populations correspond to the input for the sensor-placement methodology. Using field measurements and EDMF, candidate models have been identified and
TABLE II

INITIAL AND IDENTIFIED INTERVALS FOR MODEL PARAMETERS

\begin{tabular}{|c|c|c|}
\hline Parameters & Initial range & Identification \\
\hline \multicolumn{3}{|l|}{ Singapore Flyover } \\
\hline $\boldsymbol{\theta}_{1,1}[\mathrm{GPa}]$ & $20-42$ & $28.1-29.9$ \\
\hline $\boldsymbol{\theta}_{1,3}\left[\mathrm{~kg} / \mathrm{m}_{3}\right]$ & $1800-3000$ & $1810-1950$ \\
\hline $\boldsymbol{\theta}_{1,3}[\log (\mathrm{Nmm} / \mathrm{rad})]$ & $9-13$ & $12.2-12.8$ \\
\hline $\boldsymbol{\theta}_{\mathbf{1}, \mathbf{4}}[\log (\mathrm{N} / \mathrm{mm})]$ & $8-11$ & $8.5-8.9$ \\
\hline \multicolumn{3}{|c|}{ Exeter Bascule Bridge } \\
\hline $\boldsymbol{\theta}_{2, \mathbf{1}}[\mathrm{GPa}]$ & $60-80$ & $60.2-79.8$ \\
\hline $\boldsymbol{\theta}_{\mathbf{2 , 2}}[\log (\mathrm{Nmm} / \mathrm{rad})]$ & $8-12$ & $8.1-11.9$ \\
\hline $\boldsymbol{\theta}_{2,3}[\log (\mathrm{N} / \mathrm{mm})]$ & $3-5$ & $4.3-4.4$ \\
\hline \multicolumn{3}{|c|}{ Rockingham Bridge } \\
\hline $\boldsymbol{\theta}_{3,1}[\mathrm{GPa}]$ & $25-45$ & $25.1-44.9$ \\
\hline $\boldsymbol{\theta}_{3,2}[\mathrm{GPa}]$ & $30-50$ & $46.4-48.3$ \\
\hline
\end{tabular}

identification ranges are also shown in Table II. The aim of monitoring is to reduce as much as possible these initial ranges by falsifying model instances. Globally, parameter ranges have been drastically reduced, showing that the selection of sensor configurations by engineers was appropriate. However, some parameter-value ranges have not been significantly reduced $\left(\boldsymbol{\theta}_{2, \mathbf{1}}, \boldsymbol{\theta}_{2, \mathbf{2}}\right.$ and $\left.\boldsymbol{\theta}_{3, \mathbf{1}}\right)$ as these parameters have little impact on model-instance predictions at selected sensor locations [24-26].

To measure the information gain of the monitoring, the number of falsified model instances (falsification rate) is used instead of parameter-range reductions as the falsification rate is measured in a one-dimensional domain. This choice is made as the number of model instances is sufficient for all case studies 
to cover thoroughly parameter domains. Additionally, parameter-value identification is uninterrupted for the three bridges. Therefore, a very-high correlation is observed between the parameter-range reductions and the falsification rate.

\section{A) Singapore Flyover}
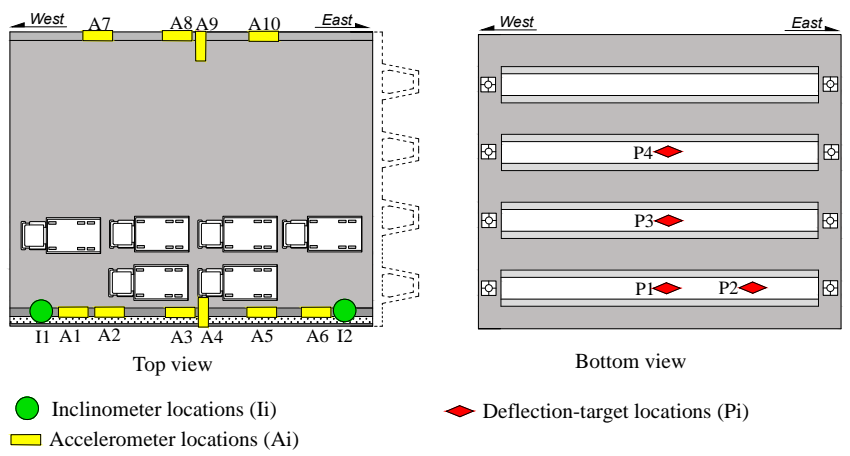

B) Exeter Bascule Bridge

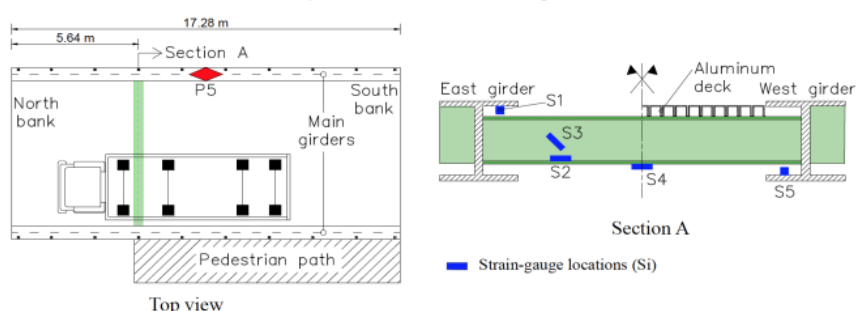

C) Rockingham Bridge

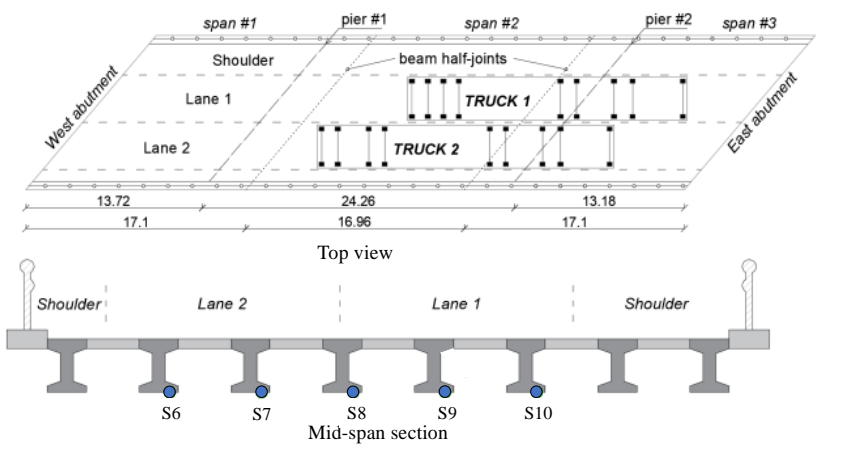

Fig. 2. Sensor positions on the bridge. (a) Singapore Flyover; (b) Exeter Bascule Bridge; (c) Rockingham Bridge.

\section{A. Sensor information entropy}

Fig. 3. presents the information entropy (left-hand side) and falsification performance (right-hand side) of each sensor for the three bridges. Each symbol shows a different type of measurements. Globally, the predicted performance is similar to the observations. Additionally, the best sensor in terms of falsification rate has always been identified by the hierarchical algorithm as the sensor with the largest information entropy for all case studies. This result shows the accuracy of the sensorplacement algorithm for prediction of independent sensor performance.

For the Exeter Bascule Bridge, S2 to S4 have entropy and falsification values equal to zero due to their locations (Fig. 2). These strain gauges are placed on a secondary beam, where model-instance predictions are little influenced by modelparameter values and thus they provide little support for
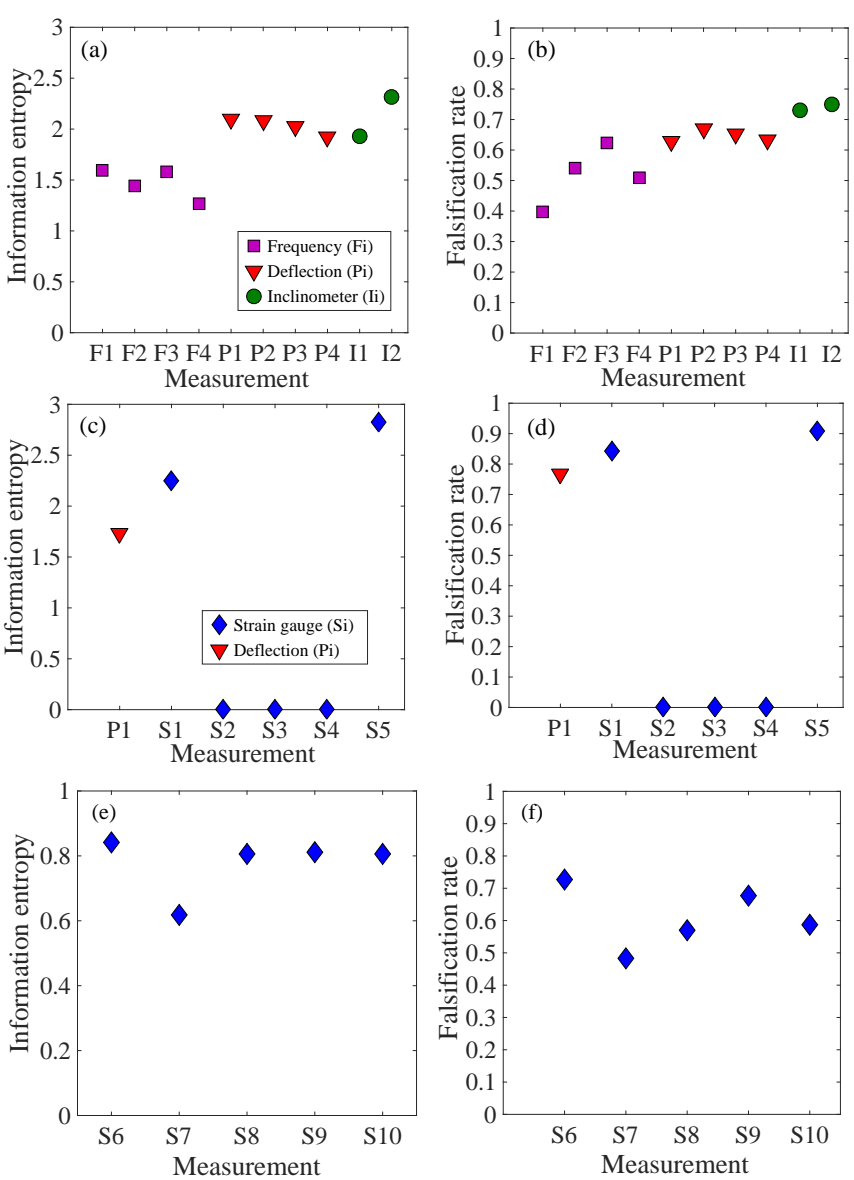

Fig. 3. Comparison of information entropy (predicted performance - (a); (c); (e)) and falsification rate (observed performance - (b); (d); (f)) for each sensor individually. (a); (b)) Singapore Flyover, (c); (d)) Exeter Bascule Bridge, (e); (f)) Rockingham Bridge.

parameter values identification. For the Rockingham Bridge, similar sensor performance is obtained because selected sensor locations are only on the midspan section of the bridge.

Table III presents the ranking correlation $\Gamma$ between falsification rate and information entropy values. Results show high correlation between the predicted and observed rankings as the $\Gamma$ value is close to 1 for each case study. These results highlight the ability of the hierarchical algorithm to predict correctly the relative individual performances of sensors among a set of possible sensor locations.

Based on the ranking correlation values of each bridge individually, a hypothesis of a correlation between the prediction and observation of sensor performances can be made. However, this result is not sufficient to validate this hypothesis because the size of data sets for each bridge individually is too low and sensor results may be correlated in

TABLE III

RANKING CORRELATION VALUE FOR EACH CASE STUDY - HIERARCHICAL ALGORITHM FOR SENSOR PLACEMENT

\begin{tabular}{cccc}
\hline $\begin{array}{c}\text { Ranking } \\
\text { correlation }\end{array}$ & $\begin{array}{c}\text { Singapore } \\
\text { Flyover }\end{array}$ & $\begin{array}{c}\text { Exeter Bascule } \\
\text { Bridge }\end{array}$ & $\begin{array}{c}\text { Rockingham } \\
\text { Bridge }\end{array}$ \\
\hline $\boldsymbol{\Gamma}[-]$ & 0.993 & 0.992 & 0.989 \\
\hline \hline
\end{tabular}


the same case study. Therefore, case-study results should be combined to perform hypothesis testing.

\section{B. Comparison of normalized bridge data}

Fig. 4. presents the relationship between informationentropy $\widehat{H}$ and falsification-rate $\widehat{F}$ values for each sensor individually. Values have been normalized in order to aggregate data from the three case studies. Additionally, the linear regression between data is shown. In case of a perfect correlation, the observed linear regression should be $\widehat{F}=\widehat{H}$.

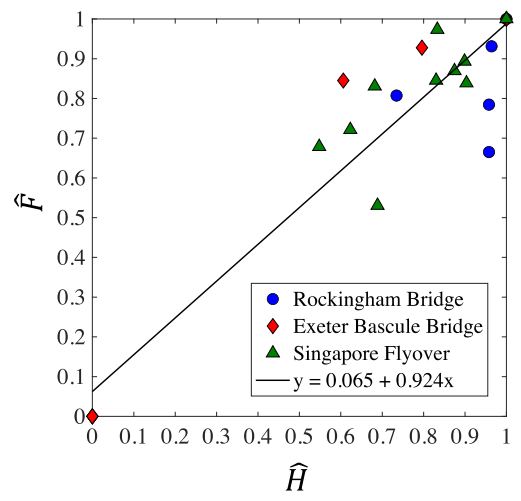

Fig. 4. Relationship between the normalized sensor information entropy $\widehat{H}$ and the normalized sensor falsification rate $\hat{F}$.

Based on the data collected, results are shown to be near to the perfect correlation with a $\mathrm{R}_{2}$ value of 0.867 . The p-value is equal to 0.01 when removing data points at $\widehat{H}=\widehat{F}=0$ to avoid extreme values in the statistical test of the p-value. These results mean that the null hypothesis of no correlation between the information entropy and the falsification performance can be a priori rejected given data sets of the three case studies. Therefore, predictions of the hierarchical algorithm in terms of predicted individual sensor performances are corroborated using field measurements.

\section{Comparison of sensor configuration information-gain assessment}

Fig. 5. presents the joint entropy (left-hand side) and falsification rate (right-hand side) with respect to the number of sensors for the three case studies. Globally, similar trends are observed between the predictions of information gain of sensor configuration with the observations. The falsification rates reach their maximum values at 9,2 and 1 sensors in the sensor configuration for the Singapore Flyover, Exeter Bascule Bridge and Rockingham Bridge, respectively, while predictions reach maximum values at 10, 3 and 4 sensors. This result verifies the validation condition specifying that the predicted information gain must reach a maximum value after the falsification rate. This condition is essential to ensure that the hierarchical algorithm is correctly estimating the unique information provided by each sensor in the sensor configuration.

Fig. 6. shows the relation between the new information metric evaluations calculated using the joint entropy predictions and falsification rate for all case studies. Sensors S2 to S4 of the Exeter Bascule Bridge (Fig. 3) are not included as they present an information-entropy value equal to zero and thus the metric values cannot be calculated. The computed linear regression between data sets is close to $N_{\text {fals }}=N_{\text {entropy }}$ with a $\mathrm{R}_{2}$ value of 0.984 . Additionally, the $\mathrm{p}$-value is smaller than 0.01 when removing data points at $N_{\text {fals }}=N_{\text {entropy }}=1$ to avoid extreme values in the statistical test of the p-value. These results corroborate the hypothesis that the hierarchical algorithm is able to predict the information gain of sensor configurations. Based on this three assessments, the hierarchical algorithm is thus recommended as sensorplacement methodology.
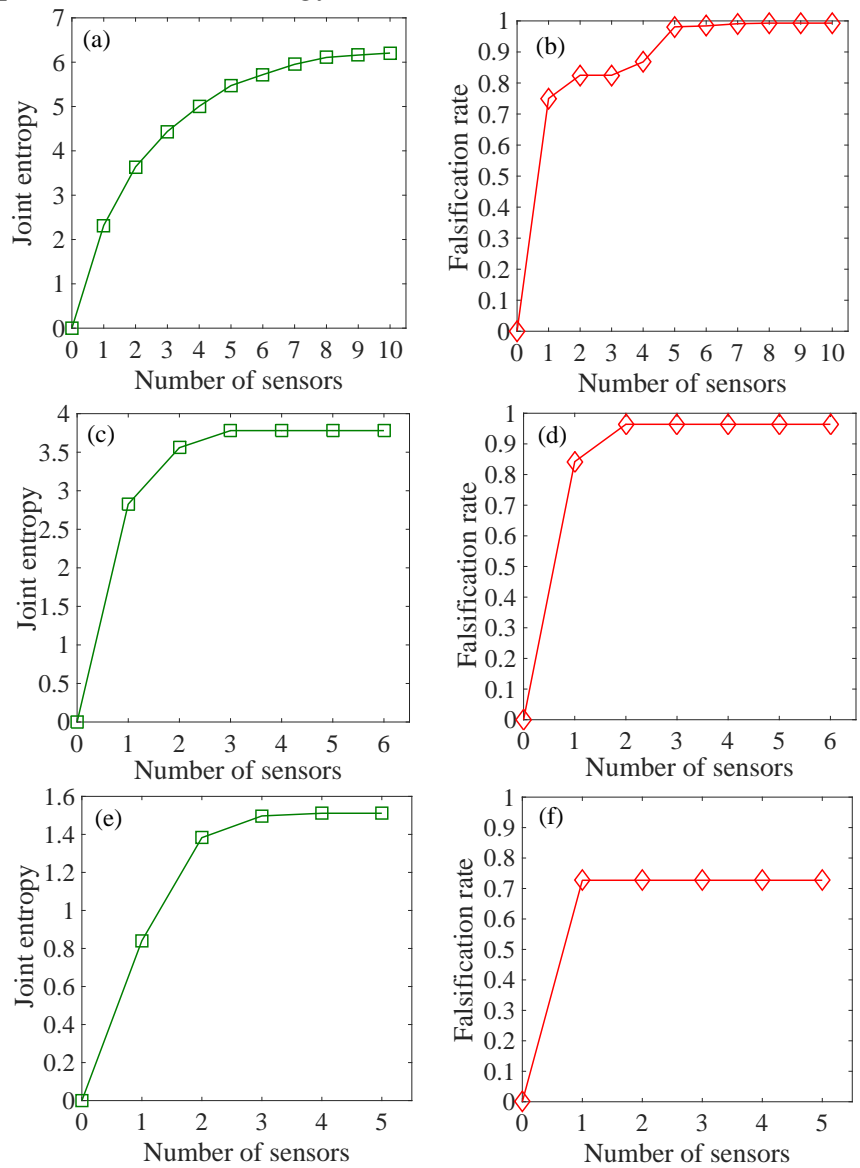

Fig. 5. Comparison of joint entropy (predicted performance - (a); (c); (e)) and falsification rate (observed performance - (b); (d); (f)) with respect to the number of sensors. (a); (b)) Singapore Flyover, (c); (d)) Exeter Bascule Bridge, (e); (f)) Rockingham Bridge.

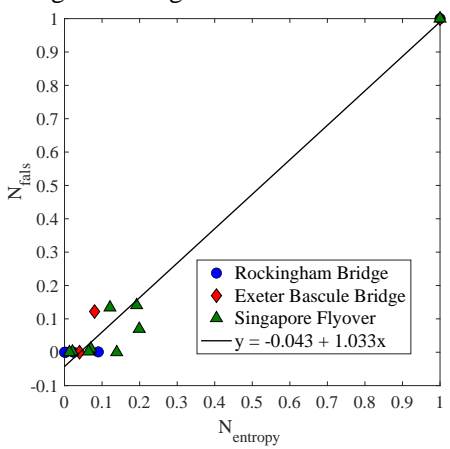

Fig. 6. Relationship between the new information metric values calculated using observations $N_{\text {fals }}$ and hierarchical-algorithm predictions $N_{\text {entropy }}$. 


\section{CASE STUDIES- SignAL-TO-NOISE RATIO}

In this Section, a sensor-placement methodology commonly used by engineers is to dispose sensors on locations that present the largest signal-to-noise ratio (SNR) (8). The proposed strategy to validate sensor-placement methodologies is employed using the three bridge case studies (Fig. 2 and 3, Tab. I and II) as for the hierarchical algorithm (Section IV).

$$
S N R=\frac{\operatorname{Mean}\left(g_{i}\right)}{U_{i, y}}
$$

First, the predicted individual sensor performance is calculated using the SNR metric for each case study. Tab. IV presents the ranking correlation $\Gamma$ between falsification-rate and SNR values. Although results are similar with the hierarchical-algorithm values (Tab. III) for the Singapore Flyover and Rockingham Bridge, the $\Gamma$ value for the Exeter Bascule Bridge is smaller than 0.80. Therefore, the first validation condition (0.95) is not satisfied (Section IIIA).

To corroborate this result, Fig. 7 presents the predicted and observed sensor performance for the Exeter Bascule Bridge. Results show that the sensor performance has been wrongly predicted by this sensor-placement strategy. This is because the SNR metric does not account for the variability of modelinstance predictions and model uncertainties.

Furthermore, the SNR metric does not provide evaluations of sensor configurations because locations are evaluated independently and thus, the third validation assessment (Section IIIC) cannot be performed. This sensor-placement strategy is not validated by field measurements and is thus not recommended.

TABLE IV

RANKING CORRELATION AND FIELD VALUE FOR EACH CASE STUDY SIGNAL-TO-NOISE RATIO

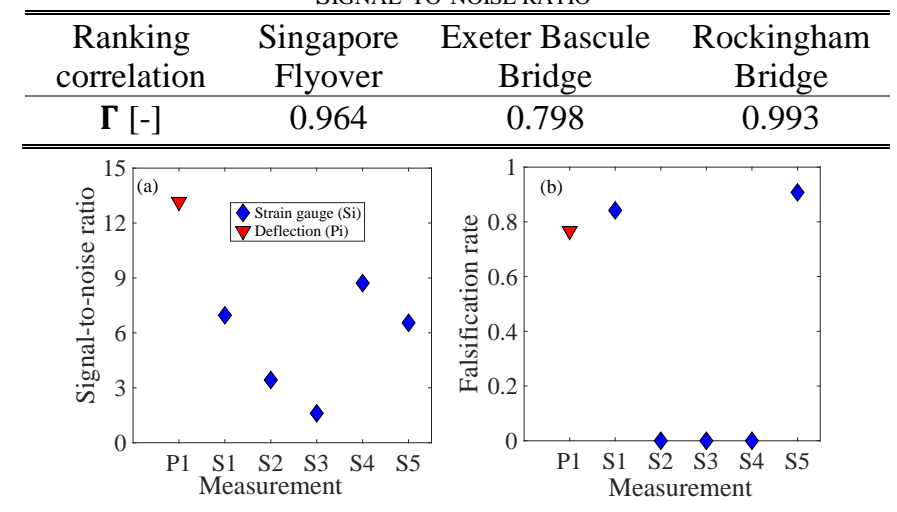

Fig. 7. Comparison of signal-to-noise ratios (predicted performance - (a)) and falsification rate (observed performance - (b)) for each sensor individually - Exeter Bascule Bridge.

\section{CONCLUSIONS}

Sensor-placement methodologies have the potential to enhance information gain when complex urban systems are monitored. These methodologies support engineers in the identification of the most powerful individual sensors as well as in the estimation of the information gain of a sensor configuration prior monitoring. Given the importance of the choice of sensor configuration for the Internet of Things, sensorplacement methodologies must be validated using field measurements. The validation of sensor-placement methodologies helps asset managers review their decision in terms of measurement-system design. Furthermore, performing a validation strategy allows the assessment of the value of monitoring information by comparing the predicted and observed information gain.

This study proposes a stepwise process to validate sensorplacement algorithms when data sets are small. The validation strategy helps reveal important outputs of the sensor-placement methodology that must be validated using field measurements. The hierarchical algorithm is shown to effectively predict the information gain provided by sensor configurations based on data sets of three full-scale case studies, while a strategy based on the signal-to-noise ratio is not recommended.

\section{ACKNOWLEDGMENT}

The research was conducted at the Future Cities Laboratory in the Singapore-ETH Centre, which was established collaboratively between ETH Zurich and Singapore's National Research Foundation (FI 370074011) under its Campus for Research Excellence and Technological Enterprise programme. The authors gratefully acknowledge the support of the Land Transport Authority (LTA) of Singapore, Main Roads Western Australia and the University of Exeter for support during load tests in the scope of the case studies.

\section{REFERENCES}

[1] I. F. C. Smith, "Studies of Sensor Data interpretation for Asset Management of the Built environment," Frontiers in Built Environment, vol. 2, pp. 2-8, 2016.

[2] F. N. Catbas, L. T. Kijewski-Correa, and A. E. Aktan, Structural identification of constructed systems. American Society of Civil Engineers, 2013.

[3] M. Proverbio, D. G. Vernay, and I. F. C. Smith, "Population-based structural identification for reservecapacity assessment of existing bridges," Journal of Civil Structural Health Monitoring, pp. 1-20, Apr. 2018.

[4] J. L. Beck and L. S. Katafygiotis, "Updating models and their uncertainties. I: Bayesian statistical framework," Journal of Engineering Mechanics, vol. 124, no. 4, pp. 455-461, 1998.

[5] L. S. Katafygiotis and J. L. Beck, "Updating models and their uncertainties. II: Model identifiability," Journal of Engineering Mechanics, vol. 124, no. 4, pp. 463-467, 1998.

[6] R. Pasquier, J.-A. Goulet, C. Acevedo, and I. F. C. Smith, "Improving fatigue evaluations of structures using inservice behavior measurement data," Journal of Bridge Engineering, vol. 19, no. 11, p. 04014045, 2014.

[7] S. G. S. Pai, A. Nussbaumer, and I. F. C. Smith, "Comparing structural identification methodologies for fatigue life prediction of a highway bridge," Frontiers in Built Environment, vol. 3, 2018.

[8] J.-A. Goulet and I. F. C. Smith, "Structural identification with systematic errors and unknown uncertainty dependencies," Computers \& Structures, vol. 128, pp. 251-258, 2013. 
[9] C. Papadimitriou, J. L. Beck, and S.-K. Au, "Entropybased optimal sensor location for structural model updating," Journal of Vibration and Control, vol. 6, no. 5, pp. 781-800, 2000.

[10] F. H. Bijarbooneh, W. Du, E. C.- Ngai, X. Fu, and J. Liu, "Cloud-assisted data fusion and sensor selection for Internet of Things," IEEE Internet of Things Journal, vol. 3, no. 3, pp. 257-268, Jun. 2016, doi: 10.1109/JIOT.2015.2502182.

[11] D. C. Kammer, "Sensor set expansion for modal vibration testing," Mechanical Systems and Signal Processing, vol. 19, no. 4, pp. 700-713, 2005.

[12] C. Papadimitriou, "Optimal sensor placement methodology for parametric identification of structural systems," Journal of sound and vibration, vol. 278, no. 4, pp. 923-947, 2004.

[13] C. Argyris, C. Papadimitriou, and P. Panetsos, "Bayesian optimal sensor placement for modal identification of civil infrastructures," Journal of Smart Cities, vol. 2, no. 2, 2017.

[14] C. Papadimitriou and G. Lombaert, "The effect of prediction error correlation on optimal sensor placement in structural dynamics," Mechanical Systems and Signal Processing, vol. 28, pp. 105-127, 2012.

[15] M. Papadopoulou, B. Raphael, I. F. C. Smith, and C. Sekhar, "Hierarchical sensor placement using joint entropy and the effect of modeling error," Entropy, vol. 16, no. 9, pp. 5078-5101, 2014.

[16] G. Z. Papadopoulos, K. Kritsis, A. Gallais, P. Chatzimisios, and T. Noel, "Performance evaluation methods in ad hoc and wireless sensor networks: a literature study," IEEE Communications Magazine, vol. 54, no. 1, pp. 122-128, Jan. 2016.

[17] C. Yang, Z. Lu, and Z. Yang, "Robust optimal sensor placement for uncertain structures with interval parameters," IEEE Sensors Journal, vol. 18, no. 5, pp. 2031-2041, 2018.

[18] F. V. C. Martins, E. G. Carrano, E. F. Wanner, R. H. C. Takahashi, and G. R. Mateus, "A hybrid multiobjective evolutionary approach for improving the performance of wireless sensor networks," IEEE Sensors Journal, vol. 11, no. 3, pp. 545-554, Mar. 2011.

[19] Y. Robert-Nicoud, B. Raphael, and I. F. C. Smith, "Configuration of measurement systems using Shannon's entropy function," Computers \& structures, vol. 83, no. 8, pp. 599-612, 2005.

[20] N. J. Bertola, M. Papadopoulou, D. Vernay, and I. F. C. Smith, "Optimal multi-type sensor placement for structural identification by static-load testing," Sensors, vol. 17, no. 12, p. 2904, 2017.

[21] M. G. Kendall, Rank Correlation Methods. Griffin, 1970.

[22] W.-J. Cao, C. G. Koh, and I. F. C. Smith, "Enhancing static-load-test identification of bridges using dynamic data," Engineering Structures, vol. 186, pp. 410-420, 2019.

[23] N. J. Bertola and I. F. C. Smith, "A methodology for measurement-system design combining information from static and dynamic excitations for bridge load testing," Journal of Sound and Vibration, vol. 463, p. 114953, Dec. 2019.

[24] M. Proverbio, N. J. Bertola, and I. F. C. Smith, "Outlierdetection methodology for structural identification using sparse static measurements," Sensors, vol. 18, no. 6, p. 1702, May 2018.

[25] N. J. Bertola, M. Cinelli, S. Casset, S. Corrente, and I. F. C. Smith, "A multi-criteria decision framework to support measurement-system design for bridge load testing," Advanced Engineering Informatics, vol. 39, pp. 186-202, Jan. 2019.

[26] N. J. Bertola and I. F. C. Smith, “A comparison of greedy and global searches for measurement-system design in bridge load testing," in SMAR 2019, Potsdam, 2019, pp. 18.

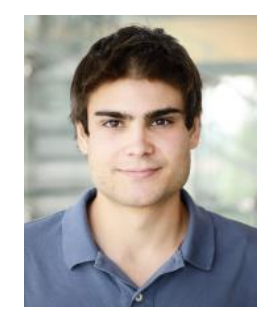

Numa J. Bertola is born in Nyon (Switzerland) in 1992. He received a B.S. (2014) and M.S. (2016) degrees in civil engineering at Ecole Polytechnique Federale de Lausanne (EPFL), Switzerland. Since 2016, he is pursuing a $\mathrm{Ph} . \mathrm{D}$. in civil engineering at the Future Cities Laboratory (FCL) in the Singapore-

ETH Centre in Singapore.

His research interests include structural health monitoring and computer science topics such as optimization and machine learning.

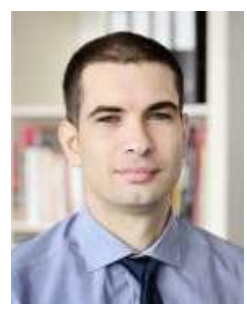

Alberto Costa is a Senior Researcher at the Singapore-ETH Centre (SEC) in Singapore. He holds B.Eng (2007) and M.Eng (2009) degrees in Computer Engineering from the University of Padova, Italy, and a Ph.D. (2012) in Operations Research from École Polytechnique, France.

His research interests include black-box and robust optimization, clustering in complex networks, and information retrieval.

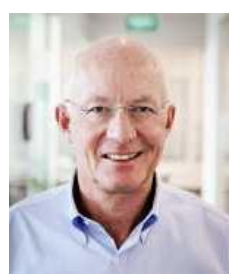

Ian F.C. Smith became a Member of IEEE in 2003. He is a Full Professor at EPFL. He received a BASc from the University of Waterloo, Canada in 1978 and a $\mathrm{PhD}$ from the University of Cambridge, UK in 1982.

His current research interests are on intersections of computer science with structures and urban systems. In 2003, he co-authored the text book Fundamentals of Computer-Aided Engineering (Wiley) and the 2nd Edition appeared in June 2013.

Prof. Smith was elected in 2004 to the Swiss Academy of Engineering Sciences and in 2005, he received the Computing in Civil Engineering Award from the ASCE. Other current appointments include Principal Investigator at the SingaporeETH Future Cities Laboratory and Adjunct Professor at Carnegie Mellon University, USA. 